\title{
Modeling of Aerosol Formation and Growth in a Laminar Flow Aerosol Generator Using Sectional Method
}

\author{
Suresh K. Varghese ${ }^{1 *}$, S. Gangamma ${ }^{2}$ \\ ${ }^{1}$ Department of Civil Engineering, National Institute of Technology Karnataka, Surathkal, \\ Karnataka, India, 575025. \\ ${ }^{2}$ Department of Chemical Engineering, National Institute of Technology Karnataka, Surathkal, \\ Karnataka, India, 575025.
}

\begin{abstract}
A model of aerosol nucleation and growth in laminar flow aerosol generator is presented. Sectional method is employed for describing aerosol dynamics. Fixed grid is used for the size structure and is compared with moving grid solution for condensation growth. Model results are compared with exact solutions in the free molecular regime. The model closely reproduces the results of exact solution for constant rate reactor. The model is also validated against the experimental results available in the literature. For the system parameters used here, the inclusion of latent heat release and Kelvin effect have not shown any impact on particle formation and growth. The effect of cooling rate on the nucleation and growth is examined using the model. The rapid cooling of the vapor-carrier gas mixture in the condenser results in highly polydispersed aerosols. The results have shown that the heat and mass transfer properties of the vapor-carrier gas system have significant effect on vapor loss to wall and the final aerosol size distribution.
\end{abstract}

Keywords: Condensation; Laminar flow aerosol generator; Monodisperse aerosols.

\footnotetext{
${ }^{*}$ Corresponding author. Tel.: +91-0824-2474604, Fax: +91-0824-2474033

E-mail address: varghesesk@gmail.com
} 


\section{INTRODUCTION}

Condensation aerosol generator has been widely used to produce monodisperse aerosol in submicron size range with a narrow size distribution of liquid droplets (Horton et al., 1991). Aerosols are produced by condensation of low vapor pressure liquids on nuclei under low flow conditions. A laminar flow system provides a fluid dynamical system which can be modeled with considerable accuracy. Since the factors that control the temperature profiles in this regime are well understood, these generators have also been used to study the kinetic process during aerosol formation and growth. These devices can be classified as laminar flow aerosol reactors.

Numerous studies on aerosol formation in steady-state condensation tube or reactor have been carried out. Early models of laminar flow reactor have been solved analytically by using Greens function approach (Davis and Liao, 1975). Pesthy et al. (1983) have done a detailed study that included homogenous nucleation and wall losses. More recent papers (Stratmann and Whitby, 1989; Wilck and Stratmann, 1997) have included variable axial velocity profiles, axial diffusion and thermophoresis deposition. The vital part of these models is the solution of the aerosol evolution and growth equations.

Often, analytical solutions are rare and limited in applicability; therefore, numerical solutions are needed. There are numerous methods with varying degrees of accuracy, and different fields of application are reported (Kumar and Ramakrishna, 1996; Zhang et al., 1999). Gelbard and Seinfeld (1980) proposed a method of sections to solve the aerosol dynamic equations numerically. Several variations of this method have been proposed (Kumar and Ramakrishna, 1997). Friedlander (1983) presented a model for nucleation and condensation constant rate reactor in the free molecular regime in terms of the first three moments of the aerosol size distribution. The moment method has been extended to the entire particle size spectrum by Pratsinis (1988) who, assuming a lognormal particle size distribution, presented a complete formulation by coupling the aerosol dynamic equation with heat and mass transport equations.

In this work we applied a modified sectional method to homogenous nucleation and heterogeneous condensation growth in a laminar flow aerosol generator. We present numerical solutions for homogeneous particle formation and comparison with analytical and experimental solutions reported in the literature. The effect of wall cooling rate on particle formation and growth is investigated with the model. The model is also used for examining the effect of transport properties of the vapor-carrier gas system on homogeneous particle formation and growth. 


\section{METHODOLOGY}

The laminar flow generators consist of four parts: nuclei generation, vapor generation, mixing and reheating, and condenser (Horton et al., 1991). Nuclei generation is carried out either by evaporation of droplets containing nonvolatile impurity, or by atomizing the salt solution. The aerosol may also be produced without the presence of seed nuclei through homogeneous nucleation. The vapor is generated by passing the carrier gas through a temperature controlled bubbler or saturator. In the reheater section proper mixing of the vapor, seed and carrier gas takes place. Also, stable inlet temperature conditions for condenser are maintained by the reheater. The condenser part of the aerosol generator, where the nuclei activation and growth occurs, is of principal interest. The model describes the system based on appropriate steady-state mass, energy and momentum conservation laws. In the following paragraphs, brief details of the model formulation is given; more detailed descriptions and discussions about assumptions can be found in Davis and Liao (1975) and Pesthy et al. (1983).

The volume fraction of the aerosol is very small. Therefore, the physical properties of the aerosol-vapor-gas mixture is taken as those of the gas. Similarly, for diffusion coefficient, D, and the thermal diffusivity, $\alpha$, we may neglect the effect of nuclei or aerosol droplets.

At low flow rates in the aerosol generator, we may write the velocity distribution as (Bird et al., 1960):

$$
V_{z}(r)=2 V_{\text {avg }}\left[1-\left(\frac{r}{R}\right)^{2}\right]
$$

where, $V_{z}(r)$ is the velocity distribution in the tube, $R$ is the radius of the tube, $V_{\text {avg }}$ is the average velocity of the cross-section of the condenser tube. Variation of the viscosity across the crosssection is neglected in the formulation (Pesthy et al., 1983). The change in flow pattern due to density changes is also not accounted for in the present model. It is assumed that the formation and growth of aerosols does not disturb the laminar velocity profile of the gas. The relationship between axial position and the residence time is:

$$
t=\frac{Z}{V_{z}(r)}
$$

In an actual experiment, it is possible that the particles formed or entered into the condenser are likely to collide with the wall by Brownian motion, and many of the particles will deposit to the wall. We have neglected both the wall loss and particle number reduction due to coagulation. 
These approximations are probably valid for low number densities used here and in experiments (Davis and Liao, 1975).

Since the condenser is cooled by heat transfer through the wall, the temperature varies in radial and axial directions. The temperature field can be described by the steady-state energy equation (Pesthy et al., 1983) as:

$$
V_{z}(r) \frac{\partial T(z, r)}{\partial z}-\frac{\alpha}{r} \frac{\partial}{\partial r}\left(r \frac{\partial T(z, r)}{\partial r}\right)=\left(\frac{\nabla H}{C_{P}}\right) \phi(z, r)
$$

where, R.H.S is the heat source, because the condensation of vapor involves the release of the heat of condensation. $\phi$ is the condensation rate and $\Delta \mathrm{H}$ is the latent heat capacity of the vapor and $\mathrm{C}_{\mathrm{p}}$ is the heat capacity of the medium. The axial conduction is ignored in the above equation; but for very small flows, the axial condition may have large effect on temperature field. The boundary conditions associated with above equation are:

$$
T(0, r)=T_{b} \quad T(z, R)=T_{w}(z)
$$

4(b)

$$
\frac{\partial T(z, 0)}{\partial r}=0
$$

where $T_{b}$ is the uniform inlet temperature and $T_{w}$ is the wall temperature profile. The various boundary conditions, fixed wall temperature and constant heat flux, can also be treated in the model.

The vapor conservation equation is written as (Pesthy et al., 1983):

$$
V_{z}(r) \frac{\partial X(z, r)}{\partial z}-\frac{D}{r} \frac{\partial}{\partial r}\left(r \frac{\partial X(z, r)}{\partial r}\right)=\phi(z, r)
$$

with boundary conditions,

$$
\begin{aligned}
& X(0, r)=x_{0} \\
& X(R, z)=\min \left(x_{0}, x^{\text {sat }}\left(T_{w}(z)\right)\right) \\
& \frac{\partial X(z, 0)}{\partial r}=0
\end{aligned}
$$


$\mathrm{X}$ is the mole fraction of the vapor, $\phi$ is the vapor sink term for condensation over the aerosols, $\mathrm{x}_{0}$ is the mole fraction of vapor entering the condenser tube, $\mathrm{T}_{\mathrm{w}}(\mathrm{z})$ is the wall temperature and $\mathrm{x}^{\text {sat }}$, is the saturation vapor mole fraction.

\section{Condensation growth and homogeneous nucleation}

If individual spherical droplets can be considered to have no relative motion with respect to the adjacent layer of fluid, the governing equation for mass transfer of vapor to a droplet can be expressed in continuum regime as (Fuchs, 1959):

$$
\frac{d D_{p}}{d t}=\frac{4 D_{v} M_{w} P_{a}}{R_{g} \rho_{p} D_{p}}\left[\frac{X_{s a t}\left(D_{p}, T_{d}\right)}{T_{d}}-\frac{X_{a}\left(T_{a}\right)}{T_{a}}\right]
$$

where, $M_{w}$ is the molecular weight of vapor, $R_{g}$ is the universal gas constant, $\rho_{p}$ is the particle density, $D_{v}$ is the diffusivity of vapor molecules, $T_{a}$ is the air temperature far from the droplet, $T_{d}$ is the droplet temperature (assumed to be uniform), $\mathrm{D}_{\mathrm{p}}$ is the droplet diameter, $\mathrm{P}_{\mathrm{a}}$ is the total pressure in the system. $X_{s a t}\left(D_{p}, T_{d}\right)$ is the equilibrium mole fraction over the particle.

The droplet equilibrium vapor pressure $\mathrm{X}_{\text {sat }}$ is calculated from flat surface vapor equilibrium by accounting for the effect of curved surface on equilibrium vapor pressure (Kelvin effect). Eq. (7) neglects the non-continuum effects that may induce error in the small droplet (Knudsen number $>>1$ ) growth estimation. The particle growth in the free molecular regime is described by (Friedlander, 2000):

$$
\frac{d D_{p}}{d t}=2 a_{c} n_{s} v_{1}(S-1)\left(\frac{k T}{2 \pi m_{1}}\right)^{\frac{1}{2}}
$$

where, $\mathrm{n}_{\mathrm{s}}$ is the molecular saturation concentration of the vapor, $\mathrm{v}_{1}$ is the volume of the monomer, $\mathrm{m}_{1}$ is the mass of the vapor monomer, $\mathrm{k}$ is Boltzmann constant, $\mathrm{S}$ is saturation ratio of the vapor and $\mathrm{T}$ is the temperature. The continuum equation is corrected by using Fuchs-Sutugin interpolation formula (Seinfeld and Pandis, 1998) for the transition regime (Fig. 1). Accommodation coefficient $\left(a_{c}\right)$ value is taken as 1 for the calculations. The equilibrium vapor pressure in Eq. (7) corresponds to the droplet surface temperature, which is corrected for heat released at the droplet surface. 


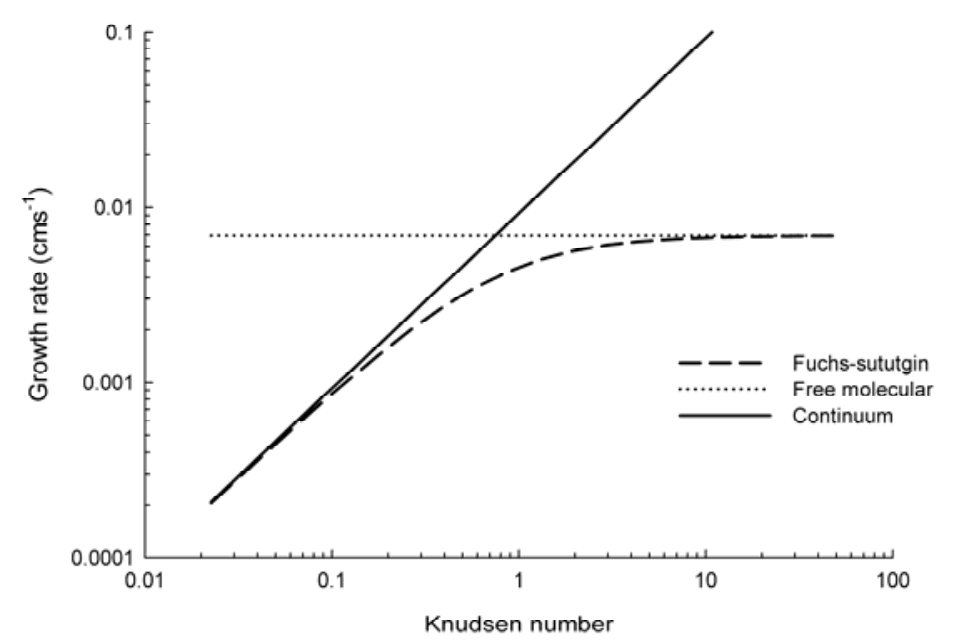

Fig. 1. Growth rate as a function of Knudsen number. Growth laws in small Knudsen number (continuum $\mathrm{Kn}<0.1$ ) and large Knudsen number (free molecular regime, $\mathrm{Kn}>10.0$ ) are given along with Fuchs-Sutugin interpolation formula.

The aerosol phase is represented by the size distribution function $N\left(D_{p}, z, r\right)$. If the number density of the droplets with diameter $\mathrm{D}_{\mathrm{p}}$ is $\mathrm{N}_{\mathrm{i}}$, then the total condensation rate per unit volume is:

$$
\phi(z, r)=\sum_{1}^{n} N_{i} \frac{1}{2} \rho_{l} \pi D_{p}{ }^{2} \frac{d D_{p}}{d t}
$$

where, $\rho_{1}$ is the density of the condensed vapor assumed as bulk liquid density.

The number of particles from the generator is dependent on the initial seed nuclei number, as well as on the homogeneous nucleation rate. While many modifications of classical theory exist, only the classical theory is employed here. Classical theory predicts the formation rate of particles of critical nucleus size from a steady-state, pre-embryo cluster distribution derived from free energy considerations. In classical theory the nucleation rate is expressed as (Pesthy et al., 1983):

$$
I=c^{*} k N^{*}
$$

where, $\mathrm{c}^{*}$ is the rate of arrival and sticking rate of monomer, $\mathrm{k}$ zeldovich non-equilibrium factor, and $\mathrm{N}^{*}$ is the concentration of the critical nuclei. The detailed description of the terms can be found in Pesthy et al. (1983) and Seinfeld and Pandis (1998). 


\section{Numerical methods}

Several numerical techniques, like method of moments (Pratsinis, 1988), orthogonal collocation (Barret and Fissan, 1989) and method of sections (Gelbard and Seinfeld, 1980) have been used to solve the general aerosol dynamic equations. Size discretised method (sectional method) has advantages when dealing with size segregated particle properties over other methods. Many variations of the sectional method are also developed and reported (Kumar and Ramakrishna, 1997). The major difference among the size discretised methods (sectional) for simulating aerosol process is the treatment of particle structure (Jacobson, 1997). The two basic structures commonly in use are full-stationary and full-moving structures. In a fixed structure the particle size distribution is divided into several size bins having different numbers of particles, but represented by same diameter and composition. When particles grow, they are moved from one size bin to another. Depending on the growth rate, the number of particles in an original size bin decreases and particle numbers in a larger size bin increase.

The disadvantage of the stationary size structure is that particle growth leads to numerical diffusion in size and to loss of information about original composition. Also, when the particle number is conserved in the growth process, it does not mean that other moments of the particle distribution are conserved. Under a full- moving grid structure, particles in each size bin grow and evaporate to their exact values, eliminating numerical diffusion during the growth (Kim and Seinfeld, 1990; Kumar and Ramakrishna, 1997). But moving grid method has disadvantages in handling transport and nucleation process. Here, a fixed structure with moving center is used for the calculation with delta function to approximate the size distribution in a bin.

A comparison of moving grid and fixed grid method for predicting particle size distribution growth is given in Fig. 2. The results are that of Dibutyl phthalate (DBP) condensation on salt seed particles $\left(10^{5} \# / \mathrm{cm}^{3}\right)$. The saturator and initial temperature was $150^{\circ} \mathrm{C}$. The temperature was linearly decreased to $27^{\circ} \mathrm{C}$ with an axial distance of $10 \mathrm{~cm}$. The result shown is the tube average particle size distribution observed at an axial distance of $12 \mathrm{~cm}$. The fixed grid with moving center gives nearly identical particle size distribution to those from full-moving structure (Jacobson, 1997). Thus, the present method reduced the numerical diffusion in the fixed grid system. The aerosol size distribution is represented using 30 sections from $0.01 \mu \mathrm{m}$ to $10 \mu \mathrm{m}$. The equations were discretised over a spatial regular grid (r) and solved using a public domain solver DVODE (Hindmarsh, 1983). The $\Delta \mathrm{r}$ and $\Delta \mathrm{z}$ were discretised to have good description of vapor and temperature field. The iteratively decided grid spacing $\Delta \mathrm{r}$ and $\Delta \mathrm{z}$ were approximately $0.05 \mathrm{~mm}$ and $1.0 \mathrm{e}^{4} / \mathrm{mm}$, respectively. 


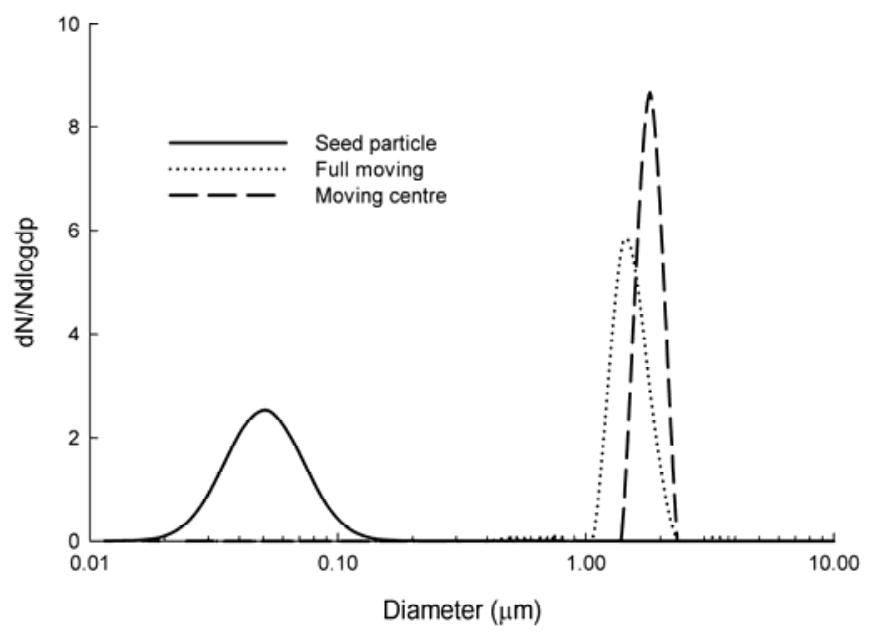

Fig. 2. Comparison of moving center and full-moving stationary size structure after condensation of DBP onto particles. The initial size distribution is shown. Results from moving center and fullmoving size structure are nearly identical.

\section{RESULTS AND DISCUSSION}

\section{Comparison with constant rate aerosol reactor}

The accuracy of the model is examined at the limiting case of aerosol dynamics for which exact results are available. A limiting case of aerosol dynamics in the free molecular regime in the absence of Kelvin effect and coagulation is presented by Friedlander (1983). For this case, the saturation-vapor concentration varied with a constant rate $(\mathrm{R})$ and the aerosol dynamics is presented by three moments of the size distribution without making assumptions for the shape of the size distribution. The details of the model can also be found in Friedlander (2000). The growth rate in free molecular regime is given by Eq. (8). The saturation ratio is governed by the external constant rate, vapor depletion by new particle formation, and condensation onto the existing particles. In the sectional formulation the saturation ratio equation becomes:

$$
\frac{d S}{d t}=R_{s}-\frac{I k^{*}}{n_{s}}-\sum_{i=1}^{n} \frac{d D_{p}}{d t} \frac{1}{2 v_{1} n_{s}} \pi D_{p i}{ }^{2} N_{i}
$$

where, $\mathrm{I}$ is the new particle generation rate given by Eq. $10, \mathrm{~N}_{\mathrm{i}}$ is the number concentration of the particles in $\mathrm{i}^{\text {th }}$ bin, $\mathrm{n}$ is the total number of size bins and $\mathrm{k}^{*}$ is the number of monomers in new particles. 


$$
k^{*}=\frac{\pi}{6}\left(\frac{4 \sum}{\ln S}\right)^{3}
$$

where, $\Sigma$ is the dimensionless surface property group (Pratsinis, 1988).

The physical parameters of the model compound used in the model are given in Table 1.

Table 1. Physical properties of the model compound (Pratsinis, 1988).

\begin{tabular}{ccc}
\hline Property & Value & Units \\
\hline $\mathrm{T}$ & 298 & $\mathrm{~K}$ \\
mol weight & 100 & $\mathrm{~g} / \mathrm{mole}$ \\
$\rho$ & 1 & $\mathrm{~g} / \mathrm{cm}^{3}$ \\
$\lambda$ & 0.065 & $\mu \mathrm{m}$ \\
$\Sigma$ & 1.85 & dimensionless \\
$\mathrm{Ns}$ & $2.43 \times 10^{8}$ & molecules $/ \mathrm{cm}^{3}$ \\
\hline
\end{tabular}

Fig. 3(a) shows the evolution of the dimensionless particle number $\left(\mathrm{N}=\Sigma \mathrm{N}_{\mathrm{i}} / \mathrm{n}_{\mathrm{s}}\right)$ concentration for a vapor saturation rate $\mathrm{R}_{\mathrm{s}}=0.1$, as predicted by sectional and moment methods described by Friedlander (1983). After the initial period, the saturation ratio in the aerosol reactor exceeds the critical value for homogenous nucleation. The particle number concentration increases rapidly with the vapor saturation ratio. The newly formed particles consume the available vapor onto their surfaces, ceasing nucleation, and particle concentration reaches an asymptotic value. The sectional model predicts satisfactorily all the features of this process.

Fig. 3(b) compares the predictions of the exact and sectional models with respect to number average particle diameter. Again, the predictions of both the models are closely similar. At the earlier stages, the particle diameter is determined by the nucleation. When the new particle formation ceases, the diameter increases due to condensation. Fig. 3(c) compares the evolution of poly-dispersity index (W) as predicted by the exact and sectional aerosol models. Although the actual values of $\mathrm{W}$ are different from the sectional model, it is clearly seen that the sectional model follows the qualitative process involved. When the new particles are formed in the reactor, large values of $\mathrm{W}$ are observed. This is because several particles with different ages are formed, resulting in the broad aerosol size distribution ( $\mathrm{W}>>0$ ). When the condensation begins, the initial size differences between particles become smaller and aerosol poly-dispersity reduced. The above limiting case shows the consistency of the sectional model used in the study and its capacity to closely reproduce the important characteristics of the product aerosol from an aerosol reactor. Therefore, the model is used for predicting the aerosol behavior in more-detailed laminar flow aerosol reactor situations. 

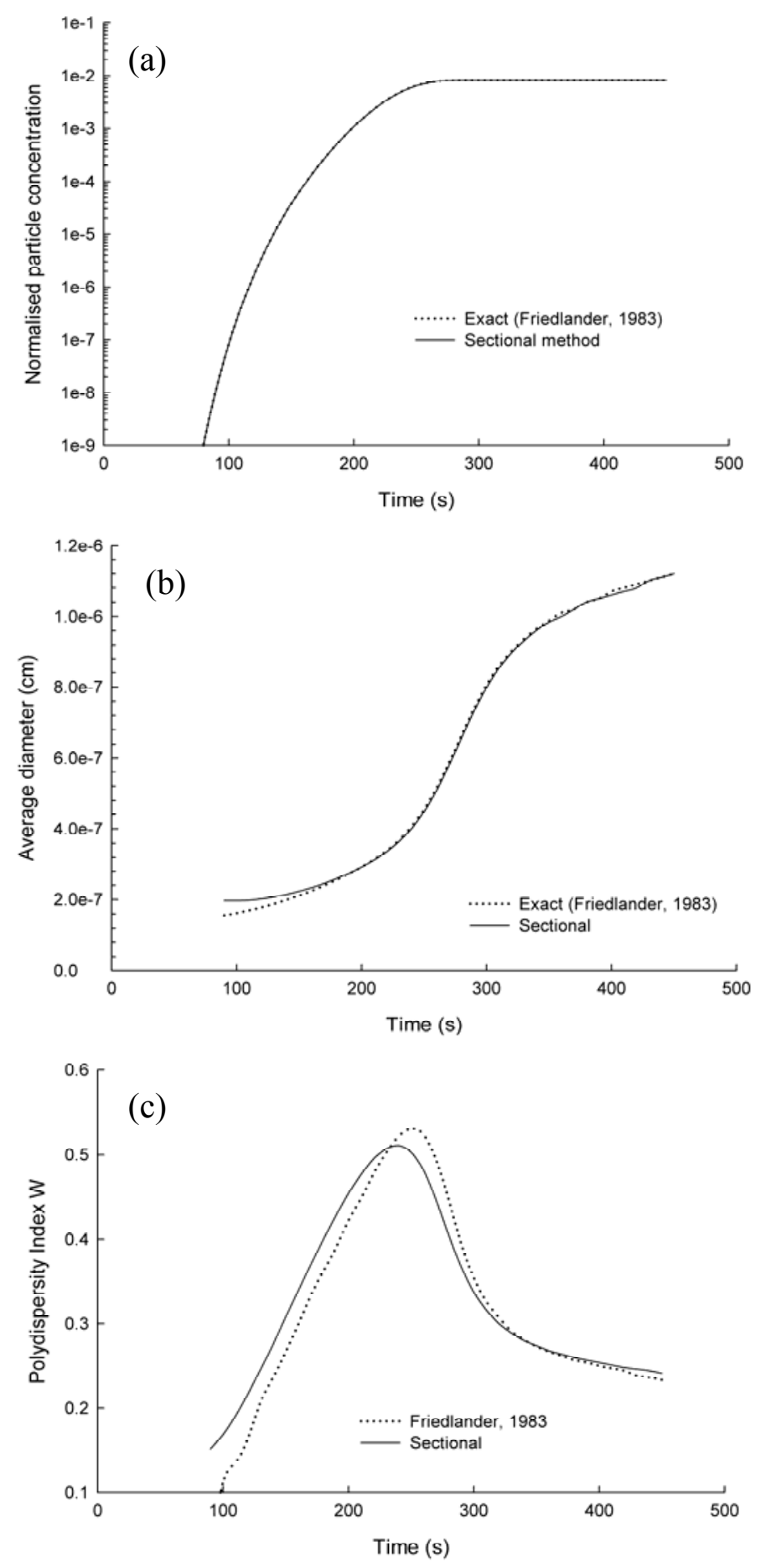

Fig. 3. Evolution of particle size distribution parameters of a constant rate batch aerosol reactor in which homogeneous nucleation and condensation is taking place. Dotted line represents exact formulation and solid line represents sectional method. (a) Particle concentration, (b) Volume average particle diameter, and (c) Polydispersed index. The sectional model closely follows the features of the exact solution. 


\section{Comparison of temperature and vapor profiles}

We now examine the predictions of the model developed above over a range of conditions reported for laminar flow aerosol generator (Nguyen et al., 1987). The above paper reported two systems for studying the homogeneous and heterogeneous nucleation in the laminar flow system. In the system, the nitrogen/air and Dibutylphthalate (DBP) was used as carrier gas and as the condensing species, respectively. The general details of the experimental system parameters are given in Table 2.

Table 2. Physical parameters of the condenser system (Nguyen, 1987).

\begin{tabular}{ccc}
\hline Parameters & System A & System B \\
\hline Tube diameter $(\mathrm{cm})$ & 1.73 & 1.0 \\
Gas flow rate $(\mathrm{L} / \mathrm{min})$ & 1.25 & $0.5,1.0$ \\
Saturator temperature $(\mathrm{K})$ & 378 & 373 \\
Condenser inlet temperature $(\mathrm{K})$ & 398 & 373 \\
Condenser final temperature & 393 & 296.7 \\
Carrier gas & Nitrogen & Dry air \\
Diffusivity of DBP $\left(\mathrm{cm}^{2} / \mathrm{s}^{1}\right)$ & $0.0398(\mathrm{~T} / 273.15)^{1.5}$ & \\
Thermal conductivity air $(\mathrm{J} / \mathrm{mKs})$ & $10^{-3}(4.39+0.071 \mathrm{~T})$ & \\
\hline
\end{tabular}

Figs. 4(a) and 4(b) show the steady state, experimental wall and centerline temperature of system B. The wall temperature is dropped rapidly to that of the cooling water temperature. As expected, the centerline temperature of the gas decreased more slowly than that of the wall. This difference is more for the larger flow rate. The model-predicted temperature profile is also given in the Figs. 4(a) and 4(b). The reproducibility of the aerosol generator profile at different wall temperature profiles was found to be very good. 

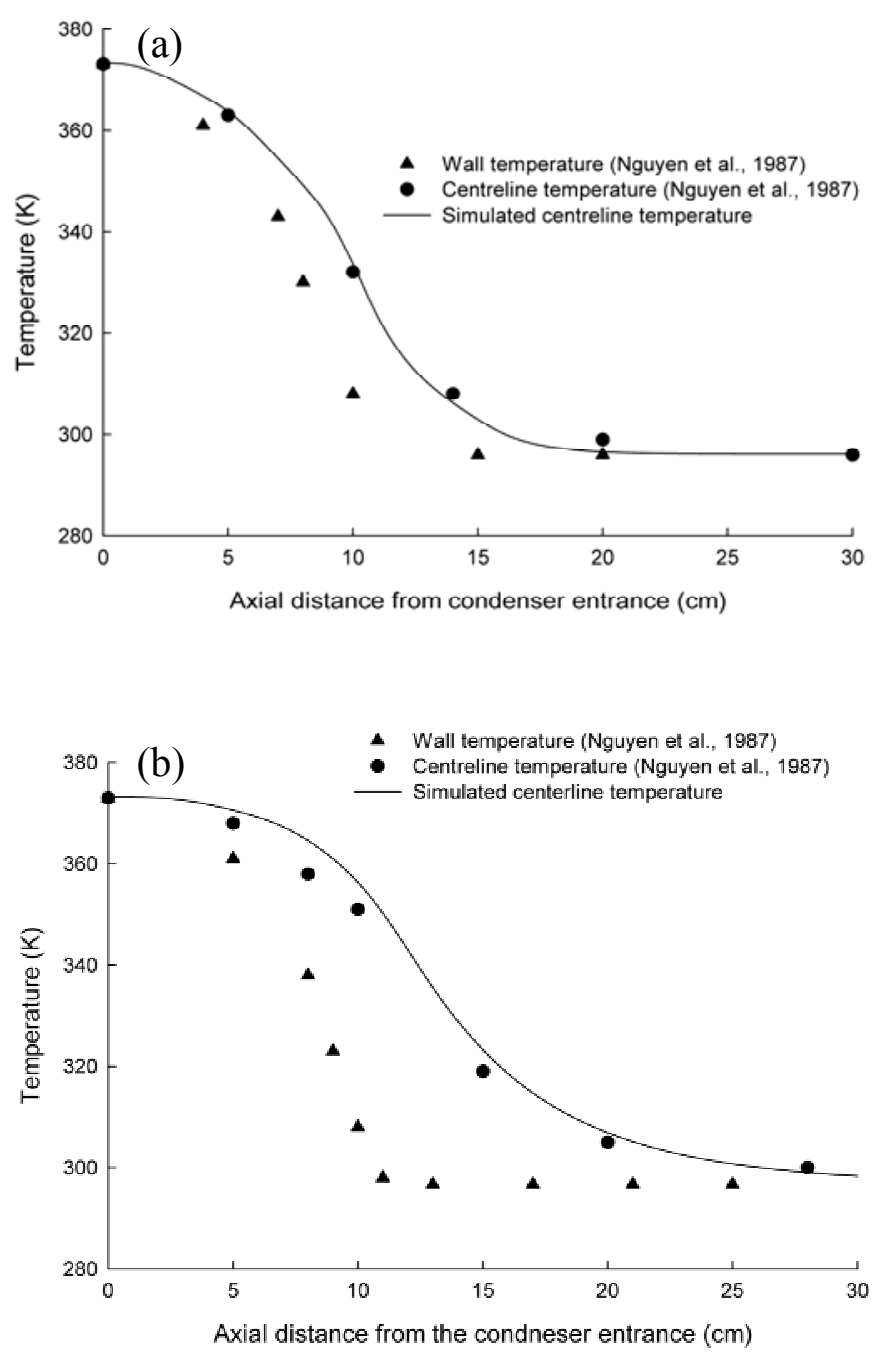

Fig. 4. Measured and calculated temperature profiles: (a) Initial temperature $373 \mathrm{~K}$, final temperature $295.8 \mathrm{~K}$, flow rate $0.5 \mathrm{~L} / \mathrm{min}$, and (b) Initial temperature $373 \mathrm{~K}$, final temperature $296.7 \mathrm{~K}$, flow rate $1.0 \mathrm{~L} / \mathrm{min}$.

Fig. 5(b) shows the dimensionless radial temperature $\left(T / T_{i}, T_{i}\right.$ is the initial condenser temperature) profile at four axial positions for the conditions given for system A without aerosol formation. The initial temperature in the condenser is $120^{\circ} \mathrm{C}$. The saturator temperature is $105^{\circ} \mathrm{C}$. The wall temperature profile is similar to that of one described in Nguyen et al. (1987) and is shown in Fig. 5(a). The non-dimension radius position ' 0 ' represents the centerline and ' 1 ' represents the wall region. As expected, the centerline temperature of the gas decreased more slowly than that of wall. Fig. 5(b) shows the normalized vapor concentration distribution in the absence of aerosol formation. The vapor concentration is decreased near the wall region due to the loss of vapor molecules to the wall. This depletion of vapor in wall region leads to radial concentration gradients and enhances the diffusion of vapor from core to wall region. In the 
absence of the aerosol formation, the vapor loss to wall and radial temperature profile determines the saturation ratio of the vapor in the tube. Fig. 5(d) shows the radial profiles of the relative saturation ratio of the vapor. To identify the movement of the saturation peak along the radial position, the saturation ratio is expressed as the ratio with the maximum saturation observed in a radial cross-section. Since the condenser is cooled through the walls, the temperature of the region near to the wall will decrease rapidly. Correspondingly, the saturation ratio first peaks near the wall. Later, the saturation ratio peak moves towards centerline. This is due to the vapor loss to the wall as well as the temperature drop front moving towards the inner core. The highest saturation ratios are achieved along the downstream centerline positions. Such features of the laminar flow condensation reactor have been reported by Davis and Liao (1975) and Pesthy et al. (1983).

The rate at which the temperature and vapor transported in the condenser is also important in determining the vapor availability for aerosol formation. The rate of mass transfer and heat transfer is compared by Lewis number (Le), which is a ratio of thermal diffusivity to mass diffusivity. The calculations presented here are based on a Lewis number $\sim 5.0$. The comparison of Figs. 4(b) and 4(c) show that the radial movement of temperature drop front was much slower than the vapor drop front movement. But, the dependence of the saturation vapor concentration of DBP on temperature is roughly exponential. Even this slow decrease in the temperature results in high super-saturation, which is enough for aerosol nucleation at the center core region. This can also be observed in the curves at axial positions of $16 \mathrm{~cm}$ and $20 \mathrm{~cm}$ in Fig. 5(c), where the saturation ratio decreases initially at the center core region relative to the wall region. This is due to the fact that there is rapid drop of temperature in the wall region. But, later the drop in temperature along with less vapor loss to wall causes the relative saturation ratio at the center core to increase. But, if the vapor diffusion rate is much faster, then there is a possibility that most of the vapor would have been lost to the wall, before nucleation takes place (Barret and Fissan, 1989).

The above discussions hold true for the region where there is no aerosol formation taking place. The formation of aerosol can modify the vapor profiles discussed above. The vapor can condense onto an existing aerosol and thus reduce the vapor deposition on to the walls. The aerosol formation due to homogeneous nucleation and subsequent condensation of vapor onto them are now described. 

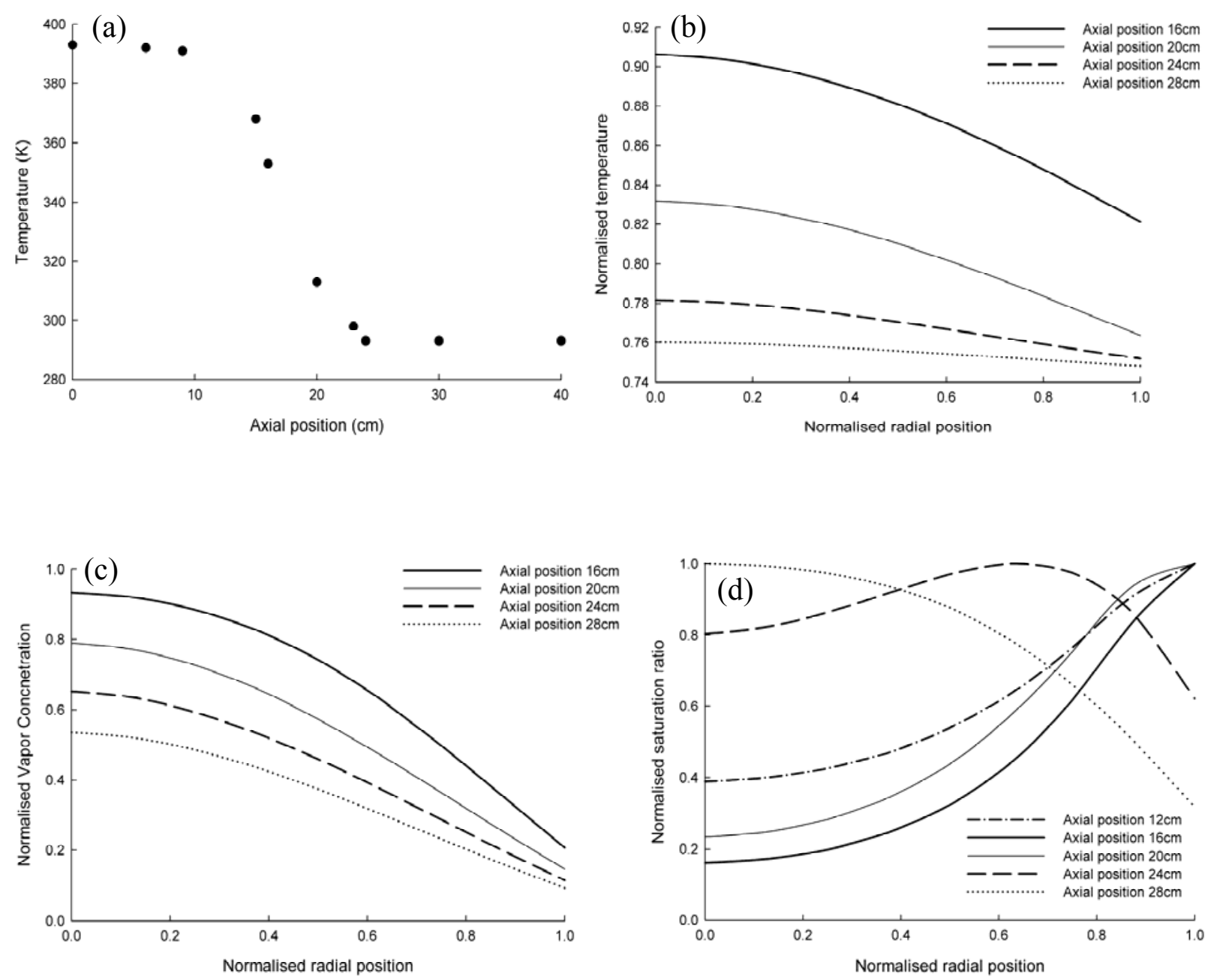

Fig. 5. (a) Wall temperature for the system (Nguyen et al., 1987). Initial temperature 393K, final temperature 293, flow rate $1.25 \mathrm{~L} / \mathrm{min}$. Radial profiles of: (b) temperature, (c) vapor concentration, and (d) vapor saturation ratio for the system.

\section{Homogeneous nucleation}

The high-saturation vapor pressure produced by the cooling in the condensation tube can provide conditions suitable for homogenous nucleation of the aerosols. The aerosols thus formed can consume the vapor by condensation and consequently reduce the vapor deposition to the wall due to the low diffusivity. The radial position of the aerosol nucleation and the effect of the presence of nucleated aerosols are discussed here. The experiments have shown that the classical nucleation theory under predicts the homogenous nucleation rate for DBP (Nguyen et al., 1987). Therefore, unless specified, an enhancement factor of $10^{5}$ for classical homogeneous nucleation is used. 
The axial variation of the homogeneous nucleation rate at various radial positions is shown in Fig. 6. The nucleation very near to the wall (the radial position $>0.9$ ) is suppressed due to vapor loss to wall (not shown in Fig. 6). But, the nucleation first appears in the region near the wall (radial position between 0.7 and 0.9 ) section and the nucleation stopped abruptly. The aerosols formed by nucleation consumed the vapor by condensation. After reaching sufficiently high concentration, the vapor scavenging by the particles stopped further nucleation. The duration of the nucleation pulse strongly depended on the growth rate of the aerosols. Similar nucleation pulses are observed at different radial positions with a time lag. But the duration and the amplitude of the nucleation pulse are also slightly different from each other. Large, duration and amplitude are found near the core region and are related to the absolute vapor availability in this region (Fig. 5c).

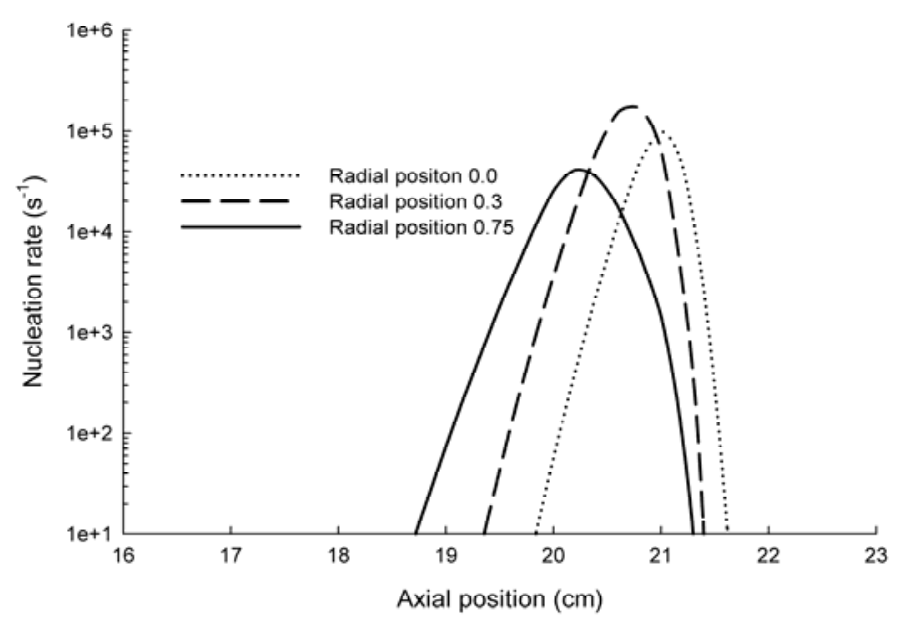

Fig. 6. Axial profiles of the nucleation rate for system A. Radial position ' 0 ' represents centerline region and ' 1 ' represents region near to wall.

Fig. 7 shows the simulated and experimentally measured (Nguyen et al., 1987) center-core averaged aerosol volume distribution for a saturator temperature of $105^{\circ} \mathrm{C}$ obtained in the system. The dashed curve is the simulated aerosol volume distribution with a classical nucleation rate enhancement factor of $10^{5}$. The simulated volume distribution curve reproduces reasonably the features of the experimental curve. The mass median diameter of the simulated aerosols is slightly larger than the experimental curve. The differences are due to the fact that the enhancement factor used may not be exactly representative of the experimental nucleation rate. Numerical experiments were carried out to examine the effect of curvature and latent heat release on the particle formation. The vapor and saturation ratio profiles vary very little between results of model runs with and without these effects. These are in accordance with the assumptions made in earlier studies (Pesthy et al., 1983). It can be stated that the model is able to perform well 
against several checks, including comparison with exact solution in the free molecular regime, temperature profiles and the experimental particle size distribution.

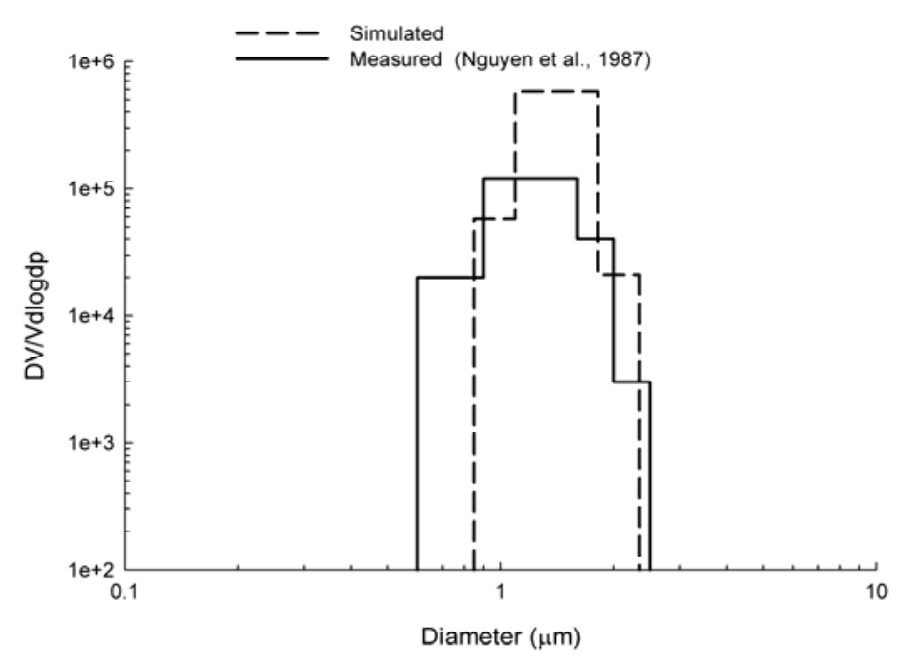

Fig. 7. Measured and simulated aerosol volume distributions generated in the system by homogeneous nucleation in the absence of seed aerosol.

\section{Effect of wall temperature profile on homogeneous nucleation}

The hot gas is cooled in the condenser tube by heat transfer through the wall. The wall cooling rate determines the temperature profile inside the condenser and further it determines the particle formation. This parameter can be controlled in a laminar flow generator. With this in mind, the effect of the wall temperature profile on particle formation is examined in this section. System A described in the above section with different wall temperature profiles is simulated, where two wall temperature profiles are considered for comparison. The initial and final wall temperatures are the same as those used above, but the wall temperature profiles are assumed to be linear with different slopes of $5^{\circ} \mathrm{C} / \mathrm{cm}$ and $20^{\circ} \mathrm{C} / \mathrm{cm}$. The radial homogeneous nucleation rate (enhanced) profiles for the two cases are depicted in Fig. 8. 


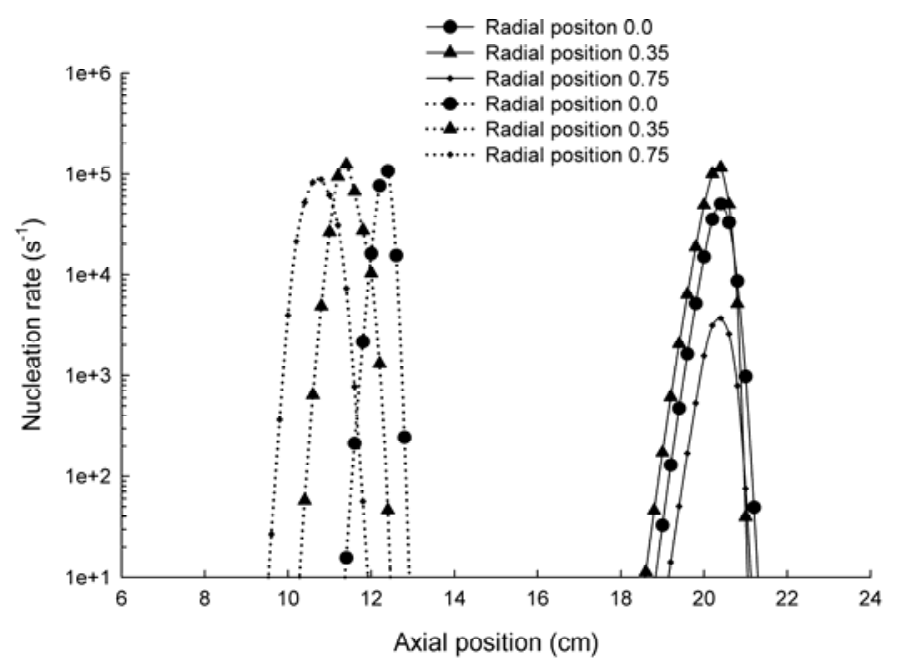

Fig. 8. Effect of wall cooling rate on homogeneous nucleation. Radial profiles of the nucleation rate for: (a) Thick line $5 \mathrm{~K} / \mathrm{cm}$ cooling, and (b) Dotted line $20 \mathrm{~K} / \mathrm{cm}$ cooling.

The thick lines represent nucleation rate with the cooling rate of $5^{\circ} \mathrm{C} / \mathrm{cm}$. The dotted lines indicate the same with the cooling rate of $20^{\circ} \mathrm{C} / \mathrm{cm}$. As expected, the axial position of the commencement of homogenous nucleation is altered with the wall cooling rate. The faster cooling rate causes rapid increase of saturation ratio of the vapor, and hence results in early beginning of nucleation. In this case, the nucleation first appears near the wall region and then moves to the center of the tube. But for the slower cooling rate (thick lines in Fig. 8), the nucleation commenced in the middle radial position and then moved to the center region and finally near to wall. These observations can be explained as follows. In the case of fast cooling, particles are formed near the wall regions. The scavenging of vapor by these particles reduces the vapor loss to the wall. But, the slow wall cooling rate causes slow initiation of particle formation. This results in loss of vapor to the wall before the particle formation begins. Such loss of vapor near the wall further leads to delayed particle formation. This is also substantiated by the low amplitude of the nucleation rate near the wall. Also, for a constant cooling rate, the axial position of the start of nucleation is also found to vary radially. This radial variation was found to be more pronounced with $20^{\circ} \mathrm{C} / \mathrm{cm}$ cooling. This may be due to the fact that large radial temperature gradients can be formed with the faster cooling rate. This consequently results in comparatively high poly-dispersity with high cooling rate.

\section{Effect of transport properties on homogeneous nucleation}

The major factor controlling particle formation and growth is the radial mass and heat transport, which is governed by the carrier vapor, system transport properties. The vapor-carrier gas system can be chosen based on the transport properties beneficial to the laminar flow generator. In this 
section, the effects of transport properties on homogenous particle formation are examined. The transport properties of the carrier gas are represented by Lewis number (Le). The system simulated here is same as in the previous section with a linear wall temperature slope of $20^{\circ} \mathrm{C} / \mathrm{cm}$. For the comparison, two cases are considered here, with Le equal to 1 and 10, respectively. The radial profiles of the nucleation rate for different Lewis number are depicted in Fig. 9.

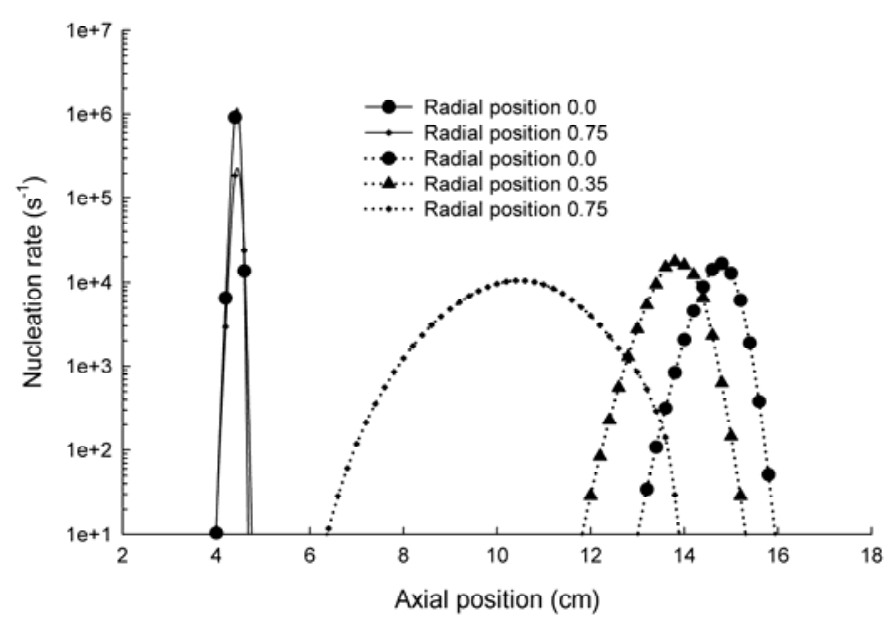

Fig. 9. Effect of mass and heat transfer rate on homogeneous nucleation. Radial profiles of the nucleation rate for: (a) Dotted line Le $=1$, and (b) Thick line Le $=10$.

The dotted curves indicate a nucleation rate with Le equal to 1. An early nucleation commencement is found for Le equal to 10. The large Lewis number represents fast temperature transfer and produces rapid cooling of the carrier gas. This creates early peaking of the saturation ratio and the particle formation. The rapid heat transfer also generates low temperature gradients along the tube cross-section. This produces more concentric nucleation-rate profiles in Fig. 9. Nearly monodispersed aerosols are formed after the growth in the high Le system. The low Le results in delayed particle formation, non-symmetric and low amplitude nucleation rate. This consequently results in very high polydispersed aerosols after the growth.

\section{Presence of seed particles on homogeneous nucleation and growth}

For homogeneous nucleation to take place, the saturation ratio of a region has to rise well above unity. If there are pre-existing particles, these particles quickly deplete the vapor and thus suppress the new particle formation. Fig. 10 shows the nucleation rate of particles with the presence of seed particle with concentration of $10^{2} / \mathrm{cm}^{2}$. The system simulated here is the same as in the previous sections, but with a linear wall temperature slope of $5^{\circ} \mathrm{C} / \mathrm{cm}$. 


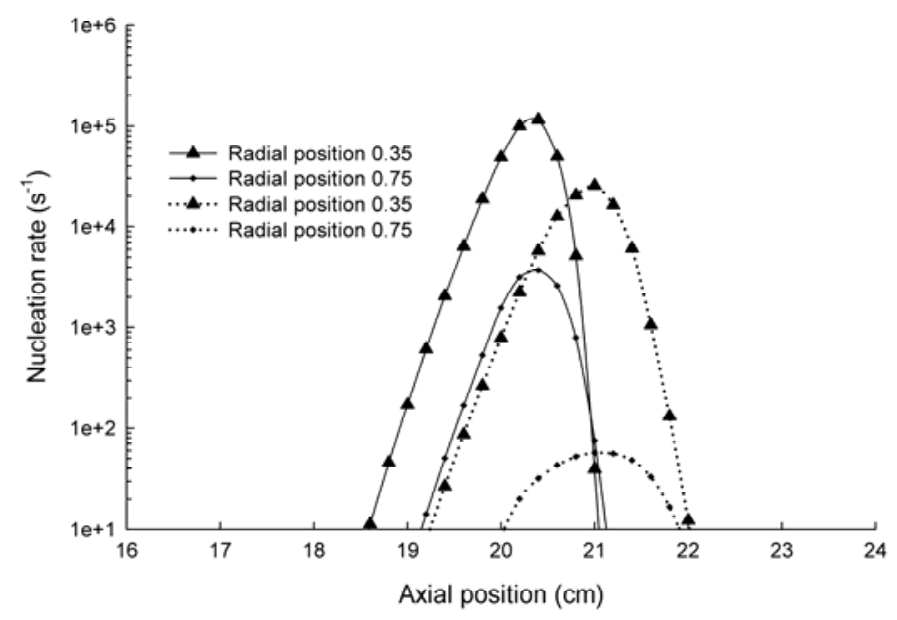

Fig. 10. Effect of seed particles on homogeneous nucleation. Radial profiles of the nucleation rate for a) Dotted line 102 particles $/ \mathrm{cm}^{3}$ b) Thick line with no particles.

The doted curves show the nucleation rate in presence of seed particles. There are two visible changes in the curves. One is the delay in the nucleation commencement and the other is the reduction in amplitude of the nucleation rate. As described above, the seed particles consume the vapor during the growth phase and thus reduce the saturation ratio of the vapor. Therefore, further cooling is necessary to increase the saturation level sufficient to produce nucleation rate. This results in delayed initiation and suppression of the new particle formation.

\section{SUMMARY AND CONCLUSION}

A sectional model for laminar flow aerosol generator, accounting for homogenous nucleation, heterogeneous nucleation and condensation, is presented. The model is applied to constant rate particle formation system (Friedlander, 1983) in the free molecular regime. The model closely predicts the integral properties of the aerosol size distribution as evidenced by comparing its predictions with exact results. The model is also compared with the experimental results. The model closely predicts properties of the temperature profiles and aerosol size distributions in a laminar flow aerosol generator.

The model is used to study the effect of cooling rate and transport properties of a vapor-carrier gas system on homogeneous particle formation. The nitrogen-DBP system is simulated for the studying the above effects. The rapid cooling of the vapor-carrier gas resulted in highly polydispersed aerosols. At the same time, a low cooling rate leads to increase in the vapor wall deposition. The high Lewis number system shows low gradients in radial temperature and 
saturation ratio profiles. Therefore, nearly monodispersed aerosols were generated for high Lewis number system.

\section{REFERENCES}

Barrett, J.C. and Fissan, H. (1989). Wall and Aerosol Condensation During Cooled Laminar Tube Flows. J. Colloid Interface Sci. 130: 498-507.

Bird, R.B., Stewart, W.E. and Lightfoot, E.N. (1960). Transport Phenomena. John Wiley and Sons, New York, p. 51.

Davis, E.J. and Liao, S.C. (1975). The Growth Kinetics and Polydispersity of Condensational Aerosols. J. Colloid Interface Sci. 50: 488-502.

Friedlander, S.K. (2000). Smoke, Dust, and Haze; Fundamentals of Aerosol Behavior. John Wiley and Sons, New York, p. 293-296.

Friedlander, S.K. (1983). Dynamics of Aerosol Formation by Chemical Reaction. Ann. NY Acad. Sci. 404: 354.

Fuchs, N.A. (1959). Evaporation and Droplet Growth in Gaseous Media. Pergamon Press. London.

Gelbard, F. and Seinfeld, J.H. (1980). Simulation of Multicomponent Aerosol Dynamics. J. Colloid Interface Sci. 78: 485-501.

Hindmarsh, A.C. (1983). ODEPACK: A Systematized Collection of Ode Solvers, Scientific Computing, edited by R. S. Stepleman., North-Holland Publishing Company, New York. p. 55.

Horton, K.D., Miller, R.D. and Mitchell, J.P. (1991). Characterization of Condensation Type Monodisperse Aerosol Generator. J. Aerosol Sci. 22: 347-363.

Jacobson, M. Z. (1997). Development and Application of a New Air Pollution Modeling SystemII. Aerosol Module Structure and Design, Atmos. Environ. 31: 131-147.

Kim, Y.P. and Seinfeld, J.H. (1990). Simulation of Multicomponent Aerosol Condensation by Moving Sectional Method. J. Colloid Interface Sci. 135: 185-199.

Kumar, S. and Ramakrishna, D. (1997). On the Solution of Population Balance Equation by Discretization - III. Nucleation, Growth and Aggregation of Particles, Chem. Eng. Sci. 52: 4659-4679.

Kumar, S. and Ramakrishna, D. (1996). On the Solution of Population Balance Equation by Discretization - I. A Fixed Pivot Technique. Chem. Eng. Sci. 51: 1311-1332.

Nguyen, H.V., Okuyama, K., Mimura, T., Kousaka, Y., Flagan, R.C. and Seinfeld, J.H. (1987). Homogeneous and Heterogeneous Nucleation in a Laminar Flow Aerosol Generator. J. Colloid Interface Sci. 119: 491-504.

Pesthy, A.J., Flagan, R.C. and Seinfeld, J.H. (1983). Theory of Aerosol Formation and Growth in Laminar Flow. J. Colloid Interface Sci. 91: 525-545. 
Pratsinis, S.E. (1988). Simultaneous Nucleation, Condensation, and Coagulation in Aerosol Reactors. J. Colloid Interface Sci. 124: 416-427.

Seinfeld, J.H. and Pandis, S.N. (1998). Atmospheric Chemistry and Physics: From Air Pollution to Climate Change. Wiley Interscience publication. New York. p. 596-607.

Stratmann, F. and Whitby, E. (1989). Heterogeneous Condensation in Cooled Laminar Tube Flow: A Comparison of Two Aerosol Modeling Techniques. J. Aerosol Sci. 20: 999-1002.

Wilck, M., and Stratmann, F. (1997). A 2-d Multicomponent Modal Aerosol Model and its Application to Laminar Flow Reactors. J. Aerosol Sci. 28: 959-972.

Zhang, Y., Seigneur, C., Seinfeld, J.H., Jacobson, M.Z. and Binkowski, F.S. (1999) Simulation of Aerosol Dynamics: A Comparative Review of Algorithms Used in Air Quality Models, Aerosol Sci. Technol. 31: 487-514.

Received for review, October 8, 2006 Accepted, December 8, 2006 